\title{
Estimation of parameters in stochastic differential equations with two random effects
}

\author{
Mohammed Sari Alsukaini*, Walaa khazaal Alkreemawi, Xiang-JunWang \\ School of Mathematics and Statistics, Huazhong University of Science and Technology, Wuhan, Hubei, 430074, P.R.China \\ *Corresponding author E-mail: mohammedsari@yahoo.com
}

\begin{abstract}
In this paper we investigate consistency and asymptotic normality of the posterior distribution of the parameters in the stochastic differential equations (SDE's) with diffusion coefficients depending nonlinearly on a random variables $\emptyset_{i}$ and $\mu_{i}$ (the random effects).The distributions of the random effects $\emptyset_{i}$ and $\mu_{i}$ depends on unknown parameters which are to be estimated from the continuous observations of the independent processes $\left(X_{i}(t), t \in\left[0, T_{i}\right], i=1, \ldots, n\right)$. We propose the Gaussian distribution for the random effect $\emptyset_{i}$ and the exponential distribution for the random effect $\mu_{i}$, we obtained an explicit formula for the likelihood function and find the estimators of the unknown parameters in the random effects.
\end{abstract}

Keywords: Stochastic Differential Equations; Maximum Likelihood Estimator; Nonlinear Random Effects; Posterior Consistency; Posterior Normality.

\section{Introduction}

Stochastic differential equations play an important role in many areas of science fields as physics, engineering, chemistry, neuroscience, biology, finance (Gugushvili and P. Spreij (2012)[8]). Statistical estimation of parameters in the diffusion processes has been studied for a long time. In the recent years, the stochastic differential equations with random effects have been the subject of diverse applications such as pharmacokinetic/pharmacodynamics, neuronal modeling (Delattre and Lavelle, 2013[4], Donnet and Samson, 2013[7], Picchini et al. 2010[13]).Maximum likelihood estimator of the parameters of the random effect, is generally not possible, because of the likelihood function is not available in most cases. Many references proposed approximations for the unknown likelihood function, for general mixed SDEs an approximations of the likelihood have been proposed (Picchini and Ditlevsen, 2011[12]), linearization (Beal and Sheiner (1982)[3]), or approximating the conditional transition density of the diffusion process given the random effects by a Hermit expansion، (AitSahalia (2002)[1]).Maitra et al. (2015) [11]) studied consistency and asymptotic normality of the posterior distribution of the parameters in the SDE's with one random effect in the drift term, Delattre et al. (2012) [6] and alkreemawi et al. (2015) [2] are studied the maximum likelihood estimator for random effects in more generally for fixed $\mathrm{T}$ and $\mathrm{n}$ tending to infinity (for non i.i.d. sample paths, see Maitra et al. (2014) [10]) and they found an explicit expression for likelihood function and exact likelihood estimator by investigate the linear random effect in the drift (multiple and additive case respectively) together with a specific distribution for the random effect. Almost researcher studied the random effect in the drift not in diffusion except Delattre and Lavelle, 2013[4] who incorporate measurement error and propose an approximation of the likelihood with the extended Kalman filter, and Delattre et al. (2014) [5] who used one random effect in the diffusion coefficient We study the consistency and asymptotic normality of the Bayesian posterior distribution when the random effects are Gaussian with a specific distributions and focus on discretely observed SDEs.

In the present work we focus on stochastic differential equation with two random effects in diffusion coefficient and suppose that the drift coefficient without random effect. We consider $\mathrm{n}$ real valued stochastic processes $\left(X_{i}(t), t \in\left[0, T_{i}\right], i=1, \ldots, n\right)$, with dynamics ruled by the following SDEs:

$$
\begin{aligned}
& d X_{i}(t)=b\left(X_{i}(t)\right) d t+\sigma\left(X_{i}(t), \emptyset_{i}, \mu_{i}\right) d W_{i}(t), X_{i}(0)=x^{i}, \\
& i=1, \ldots, n
\end{aligned}
$$

Where $W_{1}, \ldots, W_{n}$ are $n$ independent wiener processes, $\emptyset_{1}, \ldots, \emptyset_{n}$ and $\mu_{1}, \ldots, \mu_{n}$ are $n$ i.i.d. random variables taking values in ( $\mathbb{R}$ and $\mathbb{R}^{+}$) respectively, $\emptyset_{1}, \ldots, \emptyset_{n}, \mu_{1}, \ldots, \mu_{n}$ and $W_{1}, \ldots, W_{n}$ are independent and $x^{i}, i=1, \ldots, n$ are known real values. The functions $b(x)$ (drift term) and $\sigma(x)$ (diffusion term) are known real valued functions. Each process $X_{i}(t)$ represents an individual, the variables $\emptyset_{i}$ and $\mu_{i}$ represents the random effects of individual $i$, the random variables $\emptyset_{1}, \ldots, \emptyset_{n}$ have a common distribution $g(\varphi, \theta) d v(\varphi)$ on $\mathbb{R}$ and the random variables $\mu_{1}, \ldots, \mu_{n}$ have a common distribution $h(\mu, \beta) d u(\mu)$ on $\mathbb{R}^{+}$where $\theta$ and $\beta$ are an unknown parameters belonging to a set $\boldsymbol{\Theta} \subset \mathbb{R}^{p}$ where $v$ and $u$ are a dominating measures.

Our aim is to estimate $\psi=(\theta, \beta)$ from the continuous observations $\left(X_{i}(t), t \in\left[0, T_{i}\right], i=1, \ldots, n\right)$ and prove consistency and asymptotic normality of the Bayesian posterior distribution of $\psi=(\theta, \beta)$. We focus on a special case of nonlinear random effect in the diffusion coefficient in the model (1), i.e. $\sigma\left(x, \emptyset_{i}, \mu_{i}\right)=$ $\left(\emptyset_{i}+\mu_{i}\right)^{-1} \sigma(x)$, where $\sigma$ is a known real function and $\emptyset_{i}$ is a Gaussian and $\mu_{i}$ is an exponential, an explicit likelihood formula and the maximum likelihood estimator of $\psi$ are obtained.

The rest of the paper is organized as follows. Section 2 contains the notation and assumptions. The general results of the estimation of the parameters are introduced in section 3. In section 4 and exponential distribution respectively. Conclusion is given in section 5 . 


\section{Notations and assumptions}

Consider $\mathrm{n}$ real valued stochastic processes $\left(\mathrm{X}_{\mathrm{i}}(\mathrm{t}), \mathrm{t} \geq 0\right), \mathrm{i}=$ $1, \ldots, \mathrm{n}$ with dynamics ruled by (1). The processes $\mathrm{W}_{1}, \ldots, \mathrm{W}_{\mathrm{n}}$ and the random variables $\emptyset_{1}, \ldots, \emptyset_{\mathrm{n}}$ and $\mu_{1}, \ldots, \mu_{\mathrm{n}}$ are defined on a common probability space $(\Omega, \mathcal{F}, \mathbb{P})$.Consider the filtration $\left(\mathcal{F}_{\mathrm{t}}, \mathrm{t} \geq 0\right)$ defined by $\mathcal{F}_{\mathrm{t}}=\sigma\left(\emptyset_{\mathrm{i}}, \mu_{\mathrm{i}}, \mathrm{W}_{\mathrm{i}}(\mathrm{s}), \mathrm{s} \leq \mathrm{t}, \mathrm{i}=1, \ldots, \mathrm{n}\right)$. As $\quad \mathcal{F}_{\mathrm{t}}=\sigma\left(\emptyset_{\mathrm{i}}, \mu_{\mathrm{i}}, \mathrm{W}_{\mathrm{i}}(\mathrm{s}), \mathrm{s} \leq \mathrm{t}\right) \mathrm{V} \mathcal{F}_{\mathrm{t}}^{\mathrm{i}}, \quad$ with $\quad \mathcal{F}_{\mathrm{t}}^{\mathrm{i}}=$ $\sigma\left(\emptyset_{\mathrm{i}}, \emptyset_{\mathrm{j}}, \mu_{\mathrm{i}}, \mu_{\mathrm{j}}, \mathrm{W}_{\mathrm{j}}(\mathrm{s}), \mathrm{s} \leq \mathrm{t}, \mathrm{j} \neq \mathrm{i}\right)$ independent of $\mathrm{W}_{\mathrm{i}}$, each process $W_{i}$ is a $\left(\mathcal{F}_{t}, t \geq 0\right)$-Brownian motion. Moreover, the random variables $\emptyset_{\mathrm{i}}, \mu_{\mathrm{i}}$ are $\mathcal{F}_{0}$ - measurable. We assume that:

H1

i) The function $\mathrm{b}(\mathrm{x})$ is $\mathrm{C}^{1}$ on $\mathbb{R}$, and such that: $\exists \mathrm{K}>0, \forall \mathrm{x} \in \mathbb{R}, \mathrm{b}^{2}(\mathrm{x}) \leq \mathrm{K}\left(1+\mathrm{x}^{2}\right)$,

ii) The function $\sigma(\mathrm{x}, \varphi, \mu)$ is $\mathrm{C}^{1}$ on $\mathbb{R} \times \mathbb{R}^{\mathrm{d}} \times \mathbb{R}^{+}$and $\forall(\mathrm{x}, \varphi, \mu) \in \mathbb{R} \times \mathbb{R}^{\mathrm{d}} \times \mathbb{R}^{+}, \quad \sigma^{2}(\mathrm{x}, \varphi, \mu) \leq \mathrm{K}\left(1+\mathrm{x}^{2}+\right.$ $\left.|\varphi|^{2}+|\mu|^{2}\right)$.

From $\mathbf{H 1}$, the process $\left(X_{i}(t)\right)$ is well define and $\left(\varnothing_{i}, \mu_{i}, X_{i}(t)\right)$ adapted to filtration $\left(\mathcal{F}_{\mathrm{t}}, \mathrm{t} \geq 0\right)$.

The $n$ processes $\left(\emptyset_{i}, \mu_{i}, X_{i}(t), i=1, \ldots, n\right)$ are independent. For all $\varphi, \mu$ and all $x^{i} \in \mathbb{R}$, the stochastic differential equation

$$
\begin{array}{r}
d X_{i}^{\varphi, \mu}(t)=b\left(X_{i}^{\varphi, \mu}(t)\right) d t+\sigma\left(X_{i}^{\varphi, \mu}(t), \varphi, \mu\right) d W_{i}(t) \\
X_{i}^{\varphi, \mu}(0)=x^{i}
\end{array}
$$

Admits a unique strong solution process $\left(\mathrm{X}_{\mathrm{i}}^{\varphi, \mu}(\mathrm{t}), \mathrm{t} \geq 0\right)$ adapted to filtration $\left(\mathcal{F}_{t}, t \geq 0\right)$. We deduce that the conditional distribution of $X_{i}$ given $\emptyset_{i}=\varphi$ and $\mu_{i}=\mu$ identical to the distribution of $X_{i}^{\varphi, \mu}$.

\section{A general results of estimation of the pa- rameters}

\subsection{Exact likelihood}

We introduce the distribution $Q_{\varphi, \mu}^{\mathrm{x}^{\mathrm{i}}, \mathrm{T}_{\mathrm{i}}}$ of $\left(\mathrm{X}_{\mathrm{i}}^{\varphi, \mu}(\mathrm{t}), \mathrm{t} \in\left[0, \mathrm{~T}_{\mathrm{i}}\right]\right)$. Let $P_{\psi}^{\mathrm{i}}=\mathrm{g}(\varphi, \theta) \operatorname{dv}(\varphi) \otimes \mathrm{h}(\mu, \beta) \mathrm{du}(\mu) \otimes \mathrm{Q}_{\varphi}^{\mathrm{x}^{\mathrm{i}}, \mathrm{T}_{\mathrm{i}}}$ denote the joint distribution of $\left(\varnothing_{i}, \mu_{i}, X_{i}(t)\right)$ and let $Q_{\psi}^{i}$ denote the marginal distribution of $\left(X_{i}(t), t \in\left[0, T_{i}\right]\right)$. Let us consider the following assumption:

H2 For $\mathrm{i}=1, \ldots, \mathrm{n}$ and for all $\varphi, \mu, \varphi^{\prime}, \mu^{\prime}$,

$$
Q_{\varphi, \mu}^{x^{i}, T_{i}}\left(\int_{0}^{T_{i}} \frac{b^{2}\left(x_{i}^{\varphi, \mu}(t)\right)}{\sigma^{2}\left(X_{i}^{\varphi, \mu}(t), \varphi^{\prime}, \mu^{\prime}\right)} d t<+\infty\right)=1
$$

Under H1-H2, the derivative of the distribution $Q_{\varphi, \mu}^{x^{\mathrm{i}}, T_{\mathrm{i}}}$ with respect to derivative of $Q^{i}=Q_{\varphi_{0}, \mu_{0}}^{x^{i}, T_{i}}$ has the density

$$
\begin{aligned}
& \frac{\mathrm{dQ}_{\varphi, \mu}^{\mathrm{x}^{\mathrm{i}}, \mathrm{T}_{\mathrm{i}}}}{\mathrm{dQ} Q^{\mathrm{i}}}\left(\mathrm{X}_{\mathrm{i}}\right)=\mathrm{L}_{\mathrm{T}_{\mathrm{i}}}\left(\mathrm{X}_{\mathrm{i}}, \varphi, \mu\right) \\
& =\exp \left(\int_{0}^{\mathrm{T}_{\mathrm{i}}} \frac{\mathrm{b}\left(\mathrm{X}_{\mathrm{i}}(\mathrm{s})\right)}{\sigma^{2}\left(\mathrm{X}_{\mathrm{i}}(\mathrm{s}), \varphi, \mu\right)} \mathrm{dX} \mathrm{X}_{\mathrm{i}}(\mathrm{s})-\frac{1}{2} \int_{0}^{\mathrm{T}_{\mathrm{i}}} \frac{\mathrm{b}^{2}\left(\mathrm{X}_{\mathrm{i}}(\mathrm{s})\right)}{\sigma^{2}\left(\mathrm{X}_{\mathrm{i}}(\mathrm{s}), \varphi, \mu\right)} \mathrm{ds}\right)
\end{aligned}
$$

(See Liptser and Shiryaev [9]).

$\mathbf{H 3}$ for $\mathrm{i}=1, \ldots, \mathrm{n}$, we assume that $U_{i}-\frac{1}{2} V_{i}<\infty$, where:

$$
\mathrm{U}_{\mathrm{i}}=\int_{0}^{\mathrm{T}_{\mathrm{i}}} \frac{\mathrm{b}\left(\mathrm{X}_{\mathrm{i}}(\mathrm{s})\right)}{\sigma^{2}\left(\mathrm{X}_{\mathrm{i}}(\mathrm{s})\right)} \mathrm{dX} \mathrm{X}_{\mathrm{i}}(\mathrm{s}), \mathrm{V}_{\mathrm{i}}=\int_{0}^{\mathrm{T}_{\mathrm{i}}} \frac{\mathrm{b}^{2}\left(\mathrm{X}_{\mathrm{i}}(\mathrm{s})\right)}{\sigma^{2}\left(\mathrm{X}_{\mathrm{i}}(\mathrm{s})\right)} \mathrm{ds} .
$$

By independent of individuals, $P_{\psi}=\bigotimes_{i=1}^{n} P_{\psi}^{i}$ is the distribution of $\left(\emptyset_{i}, \mu_{i}, X_{i}().\right), i=1, \ldots, n$ and $Q_{\psi}=\bigotimes_{i=1}^{n} Q_{\psi}^{i}$ is the distribution of the $\operatorname{sample}\left(X_{i}(t), t \in\left[0, T_{i}\right], i=1, \ldots, n\right)$. We can compute the density of $Q_{\psi}$ w.r.t. $Q=\bigotimes_{i=1}^{n} Q^{i}$ as follow:

$\gamma_{\mathrm{i}}\left(\mathrm{X}_{\mathrm{i}}, \psi\right)=\frac{\mathrm{dQ}_{\psi}}{\mathrm{dQ}^{\mathrm{i}}}\left(\mathrm{X}_{\mathrm{i}}\right)=$ $\int_{\mathbb{R}^{+}} \int_{\mathbb{R}} \mathrm{L}_{\mathrm{T}_{\mathrm{i}}}\left(\mathrm{X}_{\mathrm{i}}, \varphi, \mu\right) \mathrm{g}(\varphi, \theta) \mathrm{h}(\mu, \beta) \mathrm{dv}(\varphi) \mathrm{du}(\mu)$

And the exact likelihood of whole sample $\left(X_{i}(t), t \in\left[0, T_{i}\right], i=\right.$ $1, \ldots, n)$ is

$\xi_{n}(\psi)=\prod_{i=1}^{n} \gamma_{i}\left(X_{i}, \psi\right)$.

\subsection{The distributions of the random effects}

Consider model (1) with nonlinear random effects in the diffusion coefficient $\sigma\left(x, \emptyset_{i}, \mu_{i}\right)=\left(\emptyset_{i}+\mu_{i}\right)^{-1} \sigma(x)$ where $\varphi \in \mathbb{R}, \mu \in \mathbb{R}^{+}$ and $b(),. \sigma($.$) are known functions. We assume that:$

$\int_{0}^{T_{i}} \frac{b^{2}\left(X_{i}(s)\right)}{\sigma^{2}\left(X_{i}(s)\right)} d s<\infty, Q_{\varphi, \mu}^{x^{i}, T_{i}}-$ a. $s$,

for all $\varphi, \mu$ and for $i=1, \ldots, n ; T_{i}=T, x^{i}=x$, so that $\left(X_{i}(t), t \in\right.$ $[0, T], i=1, \ldots, n)$ are $i . i . d$. We will use the define statistics as follow:

$\mathrm{U}_{\mathrm{i}}=\int_{0}^{\mathrm{T}} \frac{\mathrm{b}\left(\mathrm{X}_{\mathrm{i}}(\mathrm{s})\right)}{\sigma^{2}\left(\mathrm{X}_{\mathrm{i}}(\mathrm{s})\right)} \mathrm{dX} \mathrm{X}_{\mathrm{i}}(\mathrm{s}), \mathrm{V}_{\mathrm{i}}=\int_{0}^{\mathrm{T}} \frac{\mathrm{b}^{2}\left(\mathrm{X}_{\mathrm{i}}(\mathrm{s})\right)}{\sigma^{2}\left(\mathrm{X}_{\mathrm{i}}(\mathrm{s})\right)} \mathrm{ds}$

So that the density $\gamma_{\mathrm{i}}\left(\mathrm{X}_{\mathrm{i}}, \psi\right)$ is given by:

$\gamma_{\mathrm{i}}\left(\mathrm{X}_{\mathrm{i}}, \psi\right)=$

$\int_{\mathbb{R}^{+}} \int_{\mathbb{R}} \exp \left((\varphi+\mu)^{2}\left(\mathrm{U}_{\mathrm{i}}-\frac{1}{2} \mathrm{~V}_{\mathrm{i}}\right)\right) \mathrm{g}(\varphi, \theta) \mathrm{h}(\mu, \beta) \mathrm{dv}(\varphi) \mathrm{du}(\mu)$

For a general distributions, $g(\varphi, \theta) d v(\varphi)$ for the random effect $\emptyset_{i}$ and $h(\mu, \beta) d u(\mu)$ for the random effect $\mu$, it is not possible find an explicit expression for $\gamma_{i}\left(X_{i}, \psi\right)$ above, therefor we propose a specific distributions, Gaussian $\left(\lambda, \omega^{2}\right)$ for the random effect $\varphi$ and an exponential $(\beta)$ for the random effect $\mu$, which will give an explicit likelihood and then find the maximum likelihood estimators of the unknown parameters. In the next proposition an evident expression for $\gamma_{i}\left(X_{i}, \psi\right)$ is obtained when the above distributions of the random effects is with unknown parameter $\psi=\left(\lambda, \omega^{2}, \beta\right) \in$ $\mathbb{R} \times \mathbb{R}^{+} \times \mathbb{R}^{+}$. The true value is denoted by $\psi_{0}=$ $\left(\lambda_{0}, \omega^{2}{ }_{0}, \beta_{0}\right)$.

Proposition 3.1 suppose that $g(\varphi, \theta) d v(\varphi)=\mathcal{N}\left(\lambda, \omega^{2}\right)$, and $h(\mu, \beta) d u(\mu)=\exp (\beta)$ then:

$\gamma_{i}\left(X_{i}, \psi\right)=\frac{\sqrt{\pi} \beta}{\sqrt{M_{i}}} \exp \left(-\frac{1}{4} \frac{\left(\beta\left(1-2 \mathrm{M}_{i} \omega^{2}\right)-2 \lambda M_{i}\right)^{2}}{M_{i}\left(1-2 \omega^{2} M_{i}\right)}+\frac{\lambda^{2}-\lambda\left(1-2 \mathrm{M}_{i} \omega^{2}\right)}{2 \omega^{2}\left(1-2 \mathrm{M}_{\mathrm{i}} \omega^{2}\right)}\right)$

Where $M_{i}=U_{i}-\frac{1}{2} V_{i}$

Proof: from (5) we compute the joint density of $\left(\varnothing_{i}, \mu_{i}, X_{i}\right)$ :

$\exp \left((\varphi+\mu)^{2}\left(U_{i}-\frac{1}{2} V_{i}\right)\right) \frac{1}{\sqrt{2 \pi \omega^{2}}} \times \exp \left(-\frac{1}{2 \omega^{2}}(\varphi-\lambda)^{2}\right) \times$ $\beta \exp (-\beta \mu)$.

Let $M_{i}=U_{i}-\frac{1}{2} V_{i}$, then the exponent become: 


$$
D_{i}=\varphi^{2} M_{i}-\frac{1}{2 \omega^{2}}(\varphi-\lambda)^{2}+2 \varphi \mu M_{i}+\mu^{2} M_{i}-\beta \mu .
$$

We will compute the first part $\left(\varphi^{2} M_{i}-\frac{1}{2 \omega^{2}}(\varphi-\lambda)^{2}+2 \varphi \mu M_{i}\right)$ of the exponent as follow:

$\varphi^{2} M_{i}-\frac{1}{2 \omega^{2}}(\varphi-\lambda)^{2}+2 \varphi \mu M_{i}$

$=\left(M_{i}-\frac{1}{2 \omega^{2}}\right) \varphi^{2}+\left(\frac{\lambda}{\omega^{2}}+2 \mu M_{i}\right) \varphi-\frac{\lambda^{2}}{2 \omega^{2}}$

$=\frac{-1}{2}\left(\frac{1}{\omega^{2}}-2 M_{i}\right)\left(\varphi^{2}-2 \frac{\lambda+2 \omega^{2} M_{i} \mu}{1-2 \omega^{2} M_{i}} \varphi\right)-\frac{\lambda^{2}}{2 \omega^{2}}$

$=\frac{-1}{2}\left(\frac{1}{\omega^{2}}-2 M_{i}\right)\left[\left(\varphi^{2}-\frac{\lambda+2 \omega^{2} M_{i} \mu}{1-2 \omega^{2} M_{i}}\right)^{2}-\left(\frac{\lambda+2 \omega^{2} M_{i} \mu}{1-2 \omega^{2} M_{i}}\right)^{2}\right]-\frac{\lambda^{2}}{2 \omega^{2}}$

$=\frac{-1}{2}\left(\frac{1-2 \omega^{2} M_{i}}{\omega^{2}}\right)\left(\varphi-\frac{\lambda+2 \omega^{2} M_{i} \mu}{1-2 \omega^{2} M_{i}}\right)^{2}$

$+\frac{\left(\lambda+2 \omega^{2} M_{i} \mu\right)^{2}}{2 \omega^{2}\left(1-2 \omega^{2} M_{i}\right)}-\frac{\lambda^{2}}{2 \omega^{2}}$.

Now, by split the result into two parts that are independent and dependent on the random effect $\varphi$ respectively, we find that the integral of the dependent part is the integral of a Gaussian density. Then the first integral in (5) with respect to $\varphi$ yields the following result:

$$
\frac{1}{\sqrt{1-2 \omega^{2} M_{i}}} \exp \left(\frac{\left(\lambda+2 \omega^{2} M_{i} \mu\right)^{2}}{2 \omega^{2}\left(1-2 \omega^{2} M_{i}\right)}-\frac{\lambda^{2}}{2 \omega^{2}}\right)
$$

By substituting in (5), the second part of the exponent is become:

$E_{i}=\frac{\left(\lambda+2 \omega^{2} M_{i} \mu\right)^{2}}{2 \omega^{2}\left(1-2 \omega^{2} M_{i}\right)}+\mu^{2} M_{i}-\beta \mu-\frac{\lambda^{2}}{2 \omega^{2}}$

$=\left(\frac{2 \omega^{2} M_{i}^{2}}{1-2 \omega^{2} M_{i}}+M_{i}\right) \mu^{2}-\left(\beta-\frac{2 \lambda M_{i}}{1-2 \omega^{2} M_{i}}\right) \mu$

$+\frac{\lambda^{2}-\lambda\left(1-2 \omega^{2} M_{i}\right)}{2 \omega^{2}\left(1-2 \omega^{2} M_{i}\right)}$

$=\frac{M_{i}}{1-2 \omega^{2} M_{i}}\left(\mu^{2}-\left(\frac{\beta\left(1-2 \omega^{2} M_{i}\right)-2 \lambda M_{i}}{M_{i}}\right) \mu\right)$

$+\frac{\lambda^{2}-\lambda\left(1-2 \omega^{2} M_{i}\right)}{2 \omega^{2}\left(1-2 \omega^{2} M_{i}\right)}$

$=\frac{M_{i}}{1-2 \omega^{2} M_{i}}\left[\left(\mu-\frac{1}{2} \frac{\beta\left(1-2 \omega^{2} M_{i}\right)-2 \lambda M_{i}}{M_{i}}\right)^{2}-\left(\frac{1}{2} \frac{\beta\left(1-2 \omega^{2} M_{i}\right)-2 \lambda M_{i}}{M_{i}}\right)^{2}\right]$

$+\frac{\lambda^{2}-\lambda\left(1-2 \omega^{2} M_{i}\right)}{2 \omega^{2}\left(1-2 \omega^{2} M_{i}\right)}$

$=-\frac{1}{2} \frac{M_{i}}{\omega^{2} M_{i}-\frac{1}{2}}\left(\mu-\frac{1}{2} \frac{\beta\left(1-2 \omega^{2} M_{i}\right)-2 \lambda M_{i}}{M_{i}}\right)^{2}$

$-\frac{1}{4} \frac{\left(\beta\left(1-2 \omega^{2} M_{i}\right)-2 \lambda M_{i}\right)^{2}}{M_{i}\left(1-2 \omega^{2} M_{i}\right)}+\frac{\lambda^{2}-\lambda\left(1-2 \omega^{2} M_{i}\right)}{2 \omega^{2}\left(1-2 \omega^{2} M_{i}\right)}$

Now, by rearrange the second integral we see that the first part is normal depend on the random effect $\mu$ with mean is

$m_{i}=\frac{1}{2} \frac{\beta\left(1-2 \omega^{2} M_{i}\right)-2 \lambda M_{i}}{M_{i}}$,

And variance,

$\sigma_{i}^{2}=\frac{\omega^{2} M_{i}-\frac{1}{2}}{M_{i}}$,

Then, the conditional distribution of $\left(\emptyset_{i}, \mu_{i}\right)$ given $X_{i}$ is $\mathcal{N}\left(m_{i}, \sigma_{i}^{2}\right)$.

And hence,
$\gamma_{i}\left(X_{i}, \psi\right)=\frac{\sqrt{\pi} \beta}{\sqrt{M_{i}}} \exp \left(-\frac{1}{4} \frac{\left(\beta\left(1-2 M_{i} \omega^{2}\right)-2 \lambda M_{i}\right)^{2}}{M_{i}\left(1-2 \omega^{2} M_{i}\right)}+\frac{\lambda^{2}-\lambda\left(1-2 M_{i} \omega^{2}\right)}{2 \omega^{2}\left(1-2 M_{i} \omega^{2}\right)}\right)$

\subsection{The estimators of the parameters of the random ef- fects}

A natural approach to estimate $\psi=\left(\lambda, \omega^{2}, \beta\right)$ is the maximum likelihood estimation, so, the likelihood function is written as:

$\xi_{n}(\psi)=$

$\prod_{i=1}^{n} \frac{\sqrt{\pi} \beta}{\sqrt{M_{i}}} \exp \left(-\frac{1}{4} \frac{\left(\beta\left(1-2 M_{i} \omega^{2}\right)-2 \lambda M_{i}\right)^{2}}{M_{i}\left(1-2 \omega^{2} M_{i}\right)}+\frac{\left(\lambda^{2}-\lambda\left(1-2 M_{i} \omega^{2}\right)\right)}{2 \omega^{2}\left(1-2 M_{i} \omega^{2}\right)}\right)$.

And hence, the logarithm of likelihood function is,

$\mathcal{L}_{n}(\psi)=\log \xi_{n}(\psi)$

$=\log \pi^{\frac{n}{2}} \beta^{n}-\frac{1}{2} \sum_{i=1}^{n} \log \left(M_{i}\right)$

$-\sum_{i=1}^{n}\left[\frac{1}{4} \frac{\left(\beta\left(1-2 M_{i} \omega^{2}\right)-2 \lambda M_{i}\right)^{2}}{M_{i}\left(1-2 \omega^{2} M_{i}\right)}-\frac{\left(\lambda^{2}-\lambda\left(1-2 M_{i} \omega^{2}\right)\right)}{2 \omega^{2}\left(1-2 M_{i} \omega^{2}\right)}\right]$.

We will study the following score function

$G_{n}(\psi)=\left(\frac{\partial}{\partial \lambda} \mathcal{L}_{n}(\psi) \frac{\partial}{\partial \beta} \mathcal{L}_{N}(\psi) \frac{\partial}{\partial \omega^{2}} \mathcal{L}_{n}(\psi)\right)^{\prime}$

Where $x^{\prime}$ denotes the transpose of $x$, such that:

$\frac{\partial}{\partial \lambda} \mathcal{L}_{n}(\psi)=\sum_{i=1}^{n}\left[\frac{\beta\left(1-2 M_{i} \omega^{2}\right)-2 \lambda M_{i}}{1-2 \omega^{2} M_{i}}+\frac{2 \lambda-\left(1-2 M_{i} \omega^{2}\right)}{2 \omega^{2}\left(1-2 M_{i} \omega^{2}\right)}\right]$,

$=\sum_{i=1}^{n}\left[\beta+\frac{2 \lambda\left(1-2 M_{i} \omega^{2}\right)}{2 \omega^{2}\left(1-2 M_{i} \omega^{2}\right)}-\frac{1}{2 \omega^{2}}\right]$.

$=\sum_{i=1}^{n}\left[\beta+\frac{2 \lambda-1}{2 \omega^{2}}\right] \cdot \frac{\partial}{\partial \omega^{2}} \mathcal{L}_{n}(\psi)=\sum_{i=1}^{n}\left[\frac{\left(\beta\left(1-2 M_{i} \omega^{2}\right)-2 \lambda M_{i}\right) \beta}{1-2 M_{i} \omega^{2}}-\right.$

$\left.\frac{1}{2} \frac{\left(\beta\left(1-2 M_{i} \omega^{2}\right)-2 \lambda M_{i}\right)^{2}}{\left(1-2 \omega^{2} M_{i}\right)^{2}}-\frac{\lambda^{2}\left(2-8 M_{i} \omega^{2}\right)}{\left(2 \omega^{2}\left(1-2 M_{i} \omega^{2}\right)\right)^{2}}+\frac{\lambda}{2\left(\omega^{2}\right)^{2}}\right]$.

$=\sum_{i=1}^{n}\left[\frac{1}{2} \beta^{2}-\frac{2 \lambda^{2} M_{i}{ }^{2}}{\left(1-2 M_{i} \omega^{2}\right)^{2}}-\frac{2 \lambda^{2}-8 \lambda M_{i} \omega^{2}}{\left(2 \omega^{2}\left(1-2 M_{i} \omega^{2}\right)\right)^{2}}+\frac{\lambda}{2\left(\omega^{2}\right)^{2}}\right]$

$=\sum_{i=1}^{n}\left[\frac{1}{2} \beta^{2}-\frac{2 \lambda^{2} M_{i}^{2}}{\left(1-2 M_{i} \omega^{2}\right)^{2}}-\frac{2 \lambda^{2}\left(1-2 M_{i} \omega^{2}-2 M_{i} \omega^{2}\right)}{\left(2 \omega^{2}\right)^{2}\left(1-2 M_{i} \omega^{2}\right)^{2}}+\frac{\lambda}{2\left(\omega^{2}\right)^{2}}\right]$

$=$

$\sum_{i=1}^{n}\left[\frac{1}{2} \beta^{2}-\frac{2 \lambda^{2} M_{i}{ }^{2}}{\left(1-2 M_{i} \omega^{2}\right)^{2}}-\frac{2 \lambda^{2}\left(1-2 M_{i} \omega^{2}\right)}{\left(2 \omega^{2}\right)^{2}\left(1-2 M_{i} \omega^{2}\right)^{2}}+\frac{4 M_{i} \omega^{2} \lambda^{2}}{\left(2 \omega^{2}\right)^{2}\left(1-2 M_{i} \omega^{2}\right)^{2}}+\right.$ $\left.\frac{\lambda}{2\left(\omega^{2}\right)^{2}}\right]$

$=\sum_{i=1}^{n}\left[\frac{1}{2} \beta^{2}-\frac{2 \lambda^{2} M_{i}{ }^{2}}{\left(1-2 M_{i} \omega^{2}\right)^{2}}-\frac{\lambda^{2} M_{i}}{\omega^{2}\left(1-2 M_{i} \omega^{2}\right)^{2}}-\frac{2 \lambda^{2}}{\left(2 \omega^{2}\right)^{2}\left(1-2 M_{i} \omega^{2}\right)^{2}}+\right.$ $\left.\frac{\lambda}{2\left(\omega^{2}\right)^{2}}\right]$

$=\sum_{i=1}^{n}\left[\frac{1}{2} \beta^{2}+\frac{\lambda^{2} M_{i}\left(1-2 M_{i} \omega^{2}\right)}{\omega^{2}\left(1-2 M_{i} \omega^{2}\right)^{2}}-\frac{\lambda^{2}}{2\left(\omega^{2}\right)^{2}\left(1-2 M_{i} \omega^{2}\right)}+\frac{\lambda}{2\left(\omega^{2}\right)^{2}}\right]$

$=\sum_{i=1}^{n}\left[\frac{1}{2} \beta^{2}-\frac{\lambda^{2}\left(1-2 M_{i} \omega^{2}\right)}{2\left(\omega^{2}\right)^{2}\left(1-2 M_{i} \omega^{2}\right)}+\frac{\lambda}{2\left(\omega^{2}\right)^{2}}\right]$

$=\sum_{i=1}^{n}\left[\frac{1}{2} \beta^{2}-\frac{\lambda^{2}-\lambda}{2\left(\omega^{2}\right)^{2}}\right]$

$\frac{\partial}{\partial \beta} \mathcal{L}_{n}(\psi)=\sum_{i=1}^{n}\left[\frac{1}{\beta}-\frac{\beta\left(1-2 M_{i} \omega^{2}\right)}{2 M_{i}}+\lambda\right]$

$=\sum_{i=1}^{n}\left[\frac{1}{\beta}-\frac{\beta}{2 M_{i}}+\beta \omega^{2}+\lambda\right]$. 
When $\omega_{0}^{2}, \beta_{0}$ are known, the explicit estimator for $\lambda_{0}$ :

$\hat{\lambda}_{n}=\frac{2 \beta_{0} \omega_{0}^{2}-1}{2}$,

And when $\omega_{0}^{2}, \lambda_{0}$ are known, the explicit estimator for $\beta_{0}$ :

$\hat{\beta}_{n}=\left\{\begin{array}{l}\frac{2 n \lambda_{0}+\sqrt{4 n^{2} \lambda_{0}^{2}+8 n K_{i}}}{2 K_{i}} \\ \frac{2 n \lambda_{0}-\sqrt{4 n^{2} \lambda_{0}^{2}+8 n K_{i}}}{2 K_{i}}\end{array}\right.$

Where $K_{i}=\sum_{i=1}^{n} \frac{1-2 M_{i} \omega_{0}^{2}}{M_{i}}$.

When $\lambda_{0}, \beta_{0}$ are known, the explicit estimator for $\omega_{0}^{2}$ :

$\widehat{\omega_{n}^{2}}=\sqrt{\frac{\lambda_{0}^{2}-\lambda_{0}}{2 \beta_{0}^{2}}}$

Such that, $\frac{\lambda_{0}^{2}-\lambda_{0}}{2 \beta_{0}^{2}}>0$.

If all the parameters are unknown, the MLEs of $\psi_{0}=\left(\lambda_{0}, \omega_{0}^{2}, \beta_{0}\right)$ are given by the system:

$\hat{\lambda}_{n}=\frac{2 \widehat{\beta}_{n} \omega_{n}^{2}-1}{2}$,

$\hat{\beta}_{n}=\left\{\begin{array}{l}\frac{2 n \widehat{\lambda}_{n}+\sqrt{4 n^{2} \widehat{\lambda}_{n}^{2}+8 n K_{i}}}{2 K_{i}} \\ \frac{2 n \widehat{\lambda}_{n}-\sqrt{4 n^{2} \widehat{\lambda}_{n}^{2}+8 n K_{i}}}{2 K_{i}}\end{array}\right.$

$\widehat{\omega_{n}^{2}}=\sqrt{\frac{\hat{\lambda}_{n}^{2}-\widehat{\lambda}_{n}}{2{\widehat{\beta_{n}}}^{2}}}$

Such that $\frac{\widehat{\lambda}_{n}^{2}-\widehat{\lambda}_{n}}{2{\widehat{\beta_{n}}}^{2}}>0$

And $\hat{\beta}_{n}$ is a maximum likelihood estimator defined as any solution of $\mathcal{L}_{n}\left(\hat{\beta}_{n}\right)=\sup _{\psi \in \Theta} \mathcal{L}_{N}(\psi)$.

The second derivatives of $\mathcal{L}_{N}(\psi)$ with respect to the paramters is as follow:

$\frac{\partial^{2}}{\partial \lambda^{2}} \mathcal{L}_{n}(\psi)=\sum_{i=1}^{n}\left[\frac{-2 M_{i}}{1-2 \omega^{2} M_{i}}+\frac{1}{\omega^{2}\left(1-2 M_{i} \omega^{2}\right)}\right]$

$=\sum_{i=1}^{n}\left[\frac{1-2 M_{i} \omega^{2}}{\omega^{2}\left(1-2 M_{i} \omega^{2}\right)}\right]$

$=\frac{n}{\omega^{2}}$,

$\frac{\partial^{2}}{\partial \omega^{2} \partial \omega^{2}} \mathcal{L}_{n}(\psi)=\sum_{i=1}^{n} \frac{\lambda^{2}-\lambda}{\left(\omega^{2}\right)^{3}}$,

$\frac{\partial^{2}}{\partial \beta^{2}} \mathcal{L}_{n}(\psi)=\sum_{i=1}^{n}\left[2 \omega^{2}-\frac{1}{M_{i}}\right]$

$\frac{\partial^{2}}{\partial \lambda \partial \omega^{2}} \mathcal{L}_{n}(\psi)=\frac{n(1-2 \lambda)}{2\left(\omega^{2}\right)^{2}}=\frac{\partial^{2}}{\partial \omega^{2} \partial \lambda} \mathcal{L}_{N}(\psi)$

$\frac{\partial^{2}}{\partial \lambda \partial \beta} \mathcal{L}_{N}(\psi)=\frac{\partial^{2}}{\partial \beta \partial \lambda} \mathcal{L}_{N}(\psi)=n$

$\frac{\partial^{2}}{\partial \omega^{2} \partial \beta} \mathcal{L}_{n}(\psi)=\frac{\partial^{2}}{\partial \beta \partial \omega^{2}} \mathcal{L}_{n}(\psi)=n \beta$.

And the information matrix

$$
\begin{aligned}
& I(\psi)= \\
& -\left(\begin{array}{ccc}
E_{\psi}\left(\frac{\partial^{2}}{\partial \lambda^{2}} \mathcal{L}_{n}(\psi)\right) & E_{\psi}\left(\frac{\partial^{2}}{\partial \lambda \partial \omega^{2}} \mathcal{L}_{n}(\psi)\right) & E_{\psi}\left(\frac{\partial^{2}}{\partial \lambda \partial \beta} \mathcal{L}_{n}(\psi)\right) \\
E_{\psi}\left(\frac{\partial^{2}}{\partial \omega^{2} \partial \lambda} \mathcal{L}_{n}(\psi)\right) & E_{\psi}\left(\frac{\partial^{2}}{\partial \omega^{2} \partial \omega^{2}} \mathcal{L}_{n}(\psi)\right) & E_{\psi}\left(\frac{\partial^{2}}{\partial \omega^{2} \partial \beta} \mathcal{L}_{n}(\psi)\right) \\
E_{\psi}\left(\frac{\partial^{2}}{\partial \beta \partial \lambda} \mathcal{L}_{n}(\psi)\right) & E_{\psi}\left(\frac{\partial^{2}}{\partial \beta \partial \omega^{2}} \mathcal{L}_{n}(\psi)\right) & E_{\psi}\left(\frac{\partial^{2}}{\partial \beta^{2}} \mathcal{L}_{n}(\psi)\right)
\end{array}\right),
\end{aligned}
$$

Is the covariance matrix of the vector

$\left(\begin{array}{c}\frac{\partial}{\partial \lambda} \mathcal{L}_{n}(\psi) \\ \frac{\partial}{\partial \omega^{2}} \mathcal{L}_{n}(\psi) \\ \frac{\partial}{\partial \beta} \mathcal{L}_{n}(\psi)\end{array}\right)$

We need the following additional assumptions to prove the asymptotic properties:

H4 The parameter set $\Theta$ is a compact subset of $\mathbb{R} \times \mathbb{R} \times \mathbb{R}^{+}$.

H5 The true value $\psi_{0}$ belongs to $\Theta^{o}$.

H6 The matrix $I\left(\psi_{0}\right)$ is invertible.

\section{Asymptotic properties of the Bayesian pos- terior distribution}

\subsection{Consistency of the Bayesian posterior}

We consider the theorem 7.80 of schervish (1995) [14] and verify the regularity conditions in this theorem for our purpose with suppose that $\Omega$ is compact.

Theorem 1: [14]: Let $\left\{x_{n}\right\}_{n=1}^{\infty}$ be conditionally i.i.d given $\theta$ with density $f_{1}(x \mid \theta)$ with respect to a measure $v$ on a space $\left(\chi^{1}, \mathcal{B}^{1}\right)$. Fix $\theta_{o} \in \Omega$, and define, for each $M \subseteq \Omega$ and $x \in \chi^{1}$,

$Z(M, x)=\inf _{\alpha \in M} \log \frac{f_{1}\left(x \mid \theta_{o}\right)}{f_{1}(x \mid \alpha)}$

Assume that for each $\theta \neq \theta_{o}$, there is an open set $N_{\theta}$ such that $\theta \in N_{\theta}$ and that $E_{\theta_{o}} Z\left(N_{\theta}, X_{i}\right)>-\infty$.

Also assume that $f_{1}(x \mid$.) is continuous at $\theta$ for every $\theta$, a.s. $\left[P_{\theta_{o}}\right]$. For $\in>0, \operatorname{defin} C_{\in}=\left\{\theta: K_{1}\left(\theta_{o}, \theta\right)<\in\right\}$, where

$K_{1}\left(\theta_{o}, \theta\right)=E_{\theta_{o}}\left(\log \frac{f_{1}\left(X_{1} \mid \theta_{o}\right)}{f_{1}\left(X_{1} \mid \theta\right)}\right)$,

Is the kullback-leibler divergence measure associated with observation $X_{1}$.let $\pi$ be a prior distribution such that $\pi\left(C_{\epsilon}\right)>0$, for every $\in>0$.Then for every $\in>0$ and open set $N_{0}$ containing $C_{\epsilon}$, the posterior satisfies

$\lim _{n \rightarrow \infty} \pi\left(N_{0} \mid X_{1}, \ldots, X_{n}\right)=1$, a.s. $\left[P_{\theta_{o}}\right]$.

In our purpose we investigate the conditions in the theorem above as follow:

We note that for any, $f_{1}(x \mid \psi)=\gamma_{1}(x, \psi)=\gamma(x, \psi)$, Which is clearly continuous in $\psi$, so for every $\psi \neq \psi_{o}$ we get:

$\log \frac{f_{1}\left(x \mid \psi_{o}\right)}{f_{1}(x \mid \psi)}=\log \frac{\beta_{0}}{\beta}-\frac{1}{4} \frac{\left(\beta_{0}\left(1-2 \omega_{0}^{2} M_{i}\right)-2 \lambda_{0} M_{i}\right)^{2}}{M_{i}\left(1-2 \omega_{0}^{2} M_{i}\right)}$

$+\frac{\lambda_{0}^{2} \lambda_{0}\left(1-2 \omega_{0}^{2} M_{i}\right)}{2 \omega_{0}^{2}\left(1-2 \omega_{0}^{2} M_{i}\right)}+\frac{1}{4} \frac{\left(\beta\left(1-2 \omega^{2} M_{i}\right)-2 \lambda M_{i}\right)^{2}}{M_{i}\left(1-2 \omega^{2} M_{i}\right)}$

$+\frac{\lambda^{2}-\lambda\left(1-2 \omega^{2} M_{i}\right)}{2 \omega^{2}\left(1-2 \omega^{2} M_{i}\right)}$

$=\log \frac{\beta_{0}}{\beta}$ 


$$
\begin{aligned}
& -\frac{1}{4}\left(\frac{\beta_{0}^{2}\left(1-2 \omega_{0}^{2} M_{i}\right)^{2}-4 \beta_{0} \lambda_{0} M_{i}\left(1-2 \omega_{0}^{2} M_{i}\right)+4 \lambda_{0}^{2} M_{i}^{2}}{M_{i}\left(1-2 \omega_{0}^{2} M_{i}\right)}\right) \\
& +\frac{\lambda_{0}^{2}}{2 \omega_{0}^{2}\left(1-2 \omega_{0}^{2} M_{i}\right)}-\frac{\lambda_{0}}{2 \omega_{0}^{2}} \\
& +\frac{1}{4}\left(\frac{\left(\beta^{2}\left(1-2 \omega^{2} M_{i}\right)^{2}-4 \beta \lambda M_{i}\left(1-2 \omega^{2} M_{i}\right)+4 \lambda^{2} M_{i}^{2}\right)^{2}}{M_{i}\left(1-2 \omega^{2} M_{i}\right)}\right) \\
& +\frac{\lambda^{2}}{2 \omega^{2}\left(1-2 \omega^{2} M_{i}\right)}-\frac{\lambda}{2 \omega^{2}} \text {. } \\
& =\log \frac{\beta_{0}}{\beta}-\frac{1}{4} \frac{\beta_{0}^{2}\left(1-2 \omega_{0}^{2} M_{i}\right)}{M_{i}}+\beta_{0} \lambda_{0}-\frac{\lambda_{0}^{2} M_{i}}{1-2 \omega_{0}^{2} M_{i}} \\
& +\frac{\lambda_{0}^{2}}{2 \omega_{0}^{2}\left(1-2 \omega_{0}^{2} M_{i}\right)}-\frac{\lambda_{0}}{2 \omega_{0}^{2}}+\frac{1}{4} \frac{\beta^{2}\left(1-2 \omega^{2} M_{i}\right)}{M_{i}}-\beta \lambda \\
& +\frac{\lambda^{2} M_{i}}{1-2 \omega^{2} M_{i}}+\frac{\lambda^{2}}{2 \omega^{2}\left(1-2 \omega^{2} M_{i}\right)}-\frac{\lambda}{2 \omega^{2}} \\
& =\log \frac{\beta_{0}}{\beta}-\frac{1}{4}\left(\frac{\beta_{0}^{2}\left(1-2 \omega_{0}^{2} M_{i}\right)}{M_{i}}-\frac{\beta^{2}\left(1-2 \omega^{2} M_{i}\right)}{M_{i}}\right) \\
& -\left(\frac{\lambda_{0}^{2} M_{i}}{1-2 \omega_{0}^{2} M_{i}}-\frac{\lambda^{2} M_{i}}{1-2 \omega^{2} M_{i}}\right) \\
& +\left(\frac{\lambda_{0}^{2}}{2 \omega_{0}^{2}\left(1-2 \omega_{0}^{2} M_{i}\right)}+\frac{\lambda^{2}}{2 \omega^{2}\left(1-2 \omega^{2} M_{i}\right)}\right) \\
& -\left(\beta \lambda-\beta_{0} \lambda_{0}\right)-\left(\frac{\lambda_{0}}{2 \omega_{0}^{2}}-\frac{\lambda}{2 \omega^{2}}\right) \\
& =\log \frac{\beta_{0}}{\beta}-\frac{1}{4} \frac{\beta_{0}^{2}}{M_{i}}+\frac{1}{2} \omega_{0}^{2} \beta_{0}^{2}-\frac{\beta^{2}}{M_{i}}+2 \beta^{2} \omega^{2} \\
& +\frac{\lambda_{0}^{2}\left(1-2 \omega_{0}^{2} M_{i}\right)}{2 \omega_{0}^{2}\left(1-2 \omega_{0}^{2} M_{i}\right)}+\frac{\lambda^{2}\left(1-2 \omega^{2} M_{i}\right)}{2 \omega^{2}\left(1-2 \omega^{2} M_{i}\right)}-\left(\beta \lambda-\beta_{0} \lambda_{0}\right) \\
& -\left(\frac{\lambda_{0}}{2 \omega_{0}^{2}}-\frac{\lambda}{2 \omega^{2}}\right) \\
& =\log \frac{\beta_{0}}{\beta}-\frac{1}{4} \frac{\beta_{0}^{2}}{M_{i}}+\frac{1}{2} \omega_{0}^{2} \beta_{0}^{2}-\frac{\beta^{2}}{M_{i}}+2 \beta^{2} \omega^{2} \\
& -\left(\beta \lambda-\beta_{0} \lambda_{0}\right)
\end{aligned}
$$

We note that $E_{\psi_{o}} \log \frac{\beta_{0}}{\beta}, E_{\psi_{o}}\left(\frac{1}{2} \omega_{0}^{2} \beta_{0}^{2}\right), E_{\psi_{o}}\left(2 \beta^{2} \omega^{2}\right)$ and $E_{\psi_{o}}\left(\beta \lambda-\beta_{0} \lambda_{0}\right)$ are finite. Under $\mathrm{H} 3, E_{\psi_{o}}\left(\frac{1}{4} \frac{\beta_{0}^{2}}{M_{i}}\right)$ and $E_{\psi_{o}}\left(\frac{\beta^{2}}{M_{i}}\right)$ are also finite, and by assume that $N_{\psi}=(\underline{\lambda}, \bar{\lambda}) \times\left(\underline{\omega^{2}}, \overline{\omega^{2}}\right) \times(\underline{\beta}, \bar{\beta})$, follows that $E_{\psi_{o}} Z\left(N_{\psi}, X_{i}\right)>-\infty$.

Now we must prove there exists a prior $\pi$ such that it's gives positive probability to $C_{\in}$ for every $\in>0$.

As we know, the kullback equal zero if and only if $\psi=\psi_{o}$, then for any $\in>0$, the set $C_{\in}$ is non-empty provided that $\Omega \backslash\left\{\psi_{o}\right\}$ is non-empty. From the above we see that $K_{1}\left(\psi_{o}, \psi\right)$ is continuous in $\psi$ (see [6]), and since the parameter space $\Omega$ is compact , then from the properties of real analyses it is clear that $K_{1}\left(\psi_{o}, \psi\right)$ is uniformly continuous on $\Omega$, that is mean , for any $\in>0$, there exist $\delta_{\epsilon}$ such that

$\left\|\psi-\psi_{o}\right\| \leq \delta_{\epsilon}$ Implies

$\left|E_{\psi_{o}}\left(\log f_{1}\left(X_{1} \mid \psi_{o}\right)\right)-E_{\psi_{o}}\left(\log f_{1}\left(X_{1} \mid \psi\right)\right)\right|<\epsilon$,

Then, $\quad K_{1}\left(\psi_{o}, \psi\right)<\epsilon$.

Hence,

$$
\begin{aligned}
& \pi\left(C_{\epsilon}\right) \geq \pi\left(\left\{\psi:\left\|\psi-\psi_{o}\right\| \leq \delta_{\epsilon}\right\}\right) \\
& \geq\left[\inf f_{\left\{\psi:\left\|\psi-\psi_{o}\right\| \leq \delta_{\epsilon}\right\}} g(\psi)\right] \times v\left(\left\{\psi:\left\|\psi-\psi_{o}\right\| \leq \delta_{\epsilon}\right\}\right) \\
& >0 .
\end{aligned}
$$

So, we obtain that (14) holds with any prior with continuous density with respect to the Lebesgue measure.

\subsection{Asymptotic normality of the Bayesian posterior}

To verify Asymptotic normality of the posterior distribution, we investigate the conditions in the next theorem provided in ([14], Theorem 7.102).We use the notations:

$l_{n}(\theta)=\log f_{n}\left(X_{n} \mid \theta\right), l_{n}^{\prime \prime}(t)=\left(\left(\left.\frac{\partial^{2}}{\partial \theta_{i} \partial \theta_{j}} l_{n}(\theta)\right|_{\theta=t}\right)\right)$

And let

$\Sigma_{n}=\left\{\begin{array}{c}-l_{n}^{\prime \prime-1}\left(\hat{\theta}_{n}\right) \text { if the invers and } \hat{\theta}_{n} \text { exist } \\ \mathbb{1}_{d} \text { if not, }\end{array}\right.$

And $\mathbb{1}_{d}$ is the identity matrix of order $d$. Notice that $\Sigma_{n}^{-1}$ is the observed fisher information matrix. We need to investigate the following regularity conditions:

1) The parameter space is $\Omega \subseteq \mathbb{R}^{d}$ for some finite $d$.

2) $\theta_{0}$ Is a point interior to $\Omega$.

3) The prior distribution of $\Theta$ has a density with respect to Lebesgue measure that is positive and continuous at $\theta_{0}$.

4) There exists a neighborhood $N_{0} \subseteq \Omega$ of $\theta_{0}$ on which $l_{n}(\theta)=\log f\left(X_{1}, \ldots, X_{n} \mid \theta\right)$ is twice continuously differentiable with respect to all coordinates of, a.s. $\left[P_{\theta_{0}}\right]$.

Theorem 2: [15]: Let $\left\{X_{n}\right\}_{n=1}^{\infty}$ be conditionally i.i.d given $\theta$. Assume the above regularity conditions; and suppose that there exist $H_{r}(x, \theta)$ such that, for each $\theta_{0} \in \operatorname{int}(\Omega)$ and each $j$,

$\sup _{\left\|\theta-\theta_{0}\right\| \leq r}\left|\frac{\partial^{2}}{\partial \theta_{k} \partial \theta_{j}} \log f_{X_{1} \mid \Theta}\left(x \mid \theta_{0}\right)-\frac{\partial^{2}}{\partial \theta_{k} \partial \theta_{j}} \log f_{X_{1} \mid \Theta}(x \mid \theta)\right| \leq$

With,

$\lim _{r \rightarrow 0} E_{\theta_{0}} H_{r}\left(X, \theta_{0}\right)=0$.

And suppose that the conditions of theorem 1 hold, and that the fisher information matrix $I\left(\theta_{0}\right)$ is positive definite. Define $l_{n}^{\prime \prime}$ as in (15), and $\Sigma_{n}$ be defined by (16).

Let $\Gamma_{n}=\Sigma_{n}^{-\frac{1}{2}}\left(\theta-\hat{\theta}_{n}\right)$. Then for each compact subset $\mathrm{E}$ of $\mathbb{R}^{d}$ and each $\in>0$, the posterior density of $\Gamma_{n}$ given $X_{n}$ converges in probability to the standard normal distribution with density $\phi($.$) ,$ which is mean:

$\lim _{n \rightarrow \infty} P_{\theta_{0}}\left(\sup _{\Gamma \in E}\left|\pi\left(\Gamma \mid X_{1}, \ldots, X_{n}\right)-\phi(\Gamma)\right|>\epsilon\right)=0$

Now we want to investigate the regularity conditions and the conditions of the above theorem in our case:

It easy to see that the first condition in the regularity conditions is trivial. From assumption H5, the second condtion holds, and from the conditions of theorem 1 above, the third condition holds. The differentiation can be passed under the integral sign (see [7], proof of proposition 5).from(8),(9),(10),(11) and (12), we deduce that $\frac{\partial^{2}}{\partial \psi_{k} \partial \psi_{j}} \log f_{X_{1} \mid \Theta}(x \mid \psi)$ is differentiable in $\psi=\left(\lambda, \omega^{2}, \beta\right)$, that is means the fourth condition holds. From remark (3.1) , the derivatives has finite expectation, Hence (17) and (18) holds, we obtain that the information matrix $I(\psi)$ is finite, and from $\mathbf{H 6}, I(\psi)$ is invertible, hence (19) holds. 


\section{Conclusion}

We depend on SDE with random effects model framework and consider the nonlinearity assumption in the diffusion function given by $\sigma\left(x, \emptyset_{i}, \mu_{i}\right)=\left(\emptyset_{i}+\mu_{i}\right)^{-1} \sigma(x)$ where $\emptyset_{i}$ are supposed to be Gaussian random variables with mean $\lambda$ and variance $\omega^{2}$, and $\mu_{i}$ to be exponential random variables with parameter $\beta$. A closed form expression of the likelihood of the parameters of the i.i.d random effects and the maximum likelihood estimator are obtained. We proved posterior consistency and asymptotic posterior normality of the estimators by using the classical asymptotic theory of the Bayesian framework.

\section{References}

[1] Y. Aït-Sahalia, Maximum Likelihood Estimation of Discretely Sampled Diffusions: A Closed-form Approximation Approach, Econometrica 70, no. 1 (2002), 223-262. http://dx.doi.org/10.1111/1468-0262.00274.

[2] W.K, Alkreemawi, M. S. Alsukaini, and X.J. Wang, On Parameters Estimation in Stochastic Differential Equations with Additive Random Effects, journal of advances in mathematics, 11, no.3 (2015), 5018-5028.

[3] S. Beal and L. Shiner, Estimating population kinetics, Critical Reviews in Biomedical Engineering, 8 (1982), 195 - 222.

[4] M. Delattre and M. Lavielle, Coupling the SAEM algorithm and the extended Kalman filter for maximum likelihood estimation in mixed-effects diffusion models, Statistics and Its Interface, 6 (2013), 519 - 532. http://dx.doi.org/10.4310/SII.2013.v6.n4.a10

[5] M. Delattre, V. Genon-Catalot, and A. Samson, Estimation of population parameters in stochastic differential equations with random effects in the diffusion coefficient, Preprint MAP, 5 (2014), 2014 - 07.

[6] M. Delattre, V. Genon-Catalot, and A. Samson, Maximum likelihood estimation for stochastic differential equations with random effects, Scandinavian Journal of Statistics, 40 (2012), 322 - 343. http://dx.doi.org/10.1111/j.1467-9469.2012.00813.x.

[7] S. Donnet and A. Samson, A review on estimation of stochastic differential equations for pharmacokinetic-pharmacodynamics models, Advanced Drug Delivery Reviews, 65 (2013), 929 - 939. http://dx.doi.org/10.1016/j.addr.2013.03.005.

[8] S. Gugushvili and P. Spreij, Parametric inference for stochastic differential equations: a smooth and match approach, ALEA, Lat. Am. J. Probab. Math. Stat. 9 (2), (2012), 609-635.

[9] R. S. Liptser, and A. N. Shiryaev, Statistics of Random Prcesses I. General Theory, 2nd edition. Springer-Verlag, Berlin, Heidelberg, (2001).

[10] T. Maitra and S. Bhattacharya, On asymptotic related to clasical inference in stochastic differential equations with random effects, ArXiv: 1407.3968v1, (2014), 1 - 12.

[11] T. Maitra and S. Bhattacharya. On Bayesian Asymptoics in Stochastic Differential Equations with Random Effects. Statistics and Probability Letters. (2015). to appear. Avaible at "http://arxiv.org/abs/1407.3971".

[12] U. Picchini and S. Ditlevsen, Practical estimation of high dimensional stochastic differential mixed-effects models, Computational Statistics \& Data Analysis, 55 (2011), 1426 - 1444. http://dx.doi.org/10.1016/j.csda.2010.10.003.

[13] U. Picchini, A. De Gaetano, and S. Ditlevsen, Stochastic differential mixed-effects models, Scand. J. Statist., 37 (2010), $67-90$. http://dx.doi.org/10.1111/j.1467-9469.2009.00665.x.

[14] M. J. Schervish, Theory of Statistics, Springer-Verlag, New York. (1995). http://dx.doi.org/10.1007/978-1-4612-4250-5. 